\title{
Paclitaxel plus valproic acid versus paclitaxel alone as second- or third-line therapy for advanced gastric cancer: a randomized Phase II trial
}

This article was published in the following Dove Press journal:

Drug Design, Development and Therapy

25 July 2016

Number of times this article has been viewed

\author{
Sachio Fushida' \\ Jun Kinoshita' \\ Masahide Kaji ${ }^{2}$ \\ Katsunobu Oyama' \\ Yasuo Hirono ${ }^{3}$ \\ Tomoya Tsukada ${ }^{2}$ \\ Takashi Fujimura ${ }^{4}$ \\ Tetsuo Ohta' \\ On behalf of the \\ Digestive Disease Support \\ Organization Study Group \\ 'Department of Gastroenterological \\ Surgery, Kanazawa University \\ Hospital, Kanazawa, ${ }^{2}$ Department of \\ Surgery, Toyama Prefectural Central \\ Hospital, Toyama, ${ }^{3}$ First Department \\ of Surgery, Fukui University Hospital, \\ Fukui, ${ }^{4}$ Department of Surgery, Toyama \\ City Hospital, Toyama, Japan
}

Correspondence: Sachio Fushida Department of Gastroenterological Surgery, Kanazawa University Hospital, I3-I Takara-machi, Kanazawa 920-864I, Japan

Tel +8I 762652362

Fax +8I 762344260

Email fushida@staff.kanazawa-u.ac.jp
Background: Weekly paclitaxel (wPTX) is the preferred second-line chemotherapy for gastric cancer in Japan. Histone deacetylase inhibitors have been shown to decrease proliferation through cell-cycle arrest, differentiation, and apoptosis in gastric cancer cells. One histone deacetylase inhibitor, valproic acid (VPA), also inhibits tumor growth by inducing apoptosis and enhances the efficacy of paclitaxel (PTX), shown in a murine gastric cancer model. This Phase II trial was designed to evaluate the benefits of adding VPA to wPTX in patients with gastric cancer refractory to first-line treatment with fluoropyrimidine.

Patients and methods: The patients were randomly assigned in a 1:1 ratio to receive PTX $80 \mathrm{mg} / \mathrm{m}^{2}$ intravenously on days 1,8 , and 15 , every 4 weeks, or a dose of PTX plus VPA taken everyday at $7.5 \mathrm{mg} / \mathrm{kg}$ twice daily. Random assignment was carried out at the data center with a minimization method adjusted by the Eastern Cooperative Oncology Group performance status (0-1 vs 2), prior chemotherapy (first-line vs second-line), and measurable lesions (presence vs absence). The primary end point was the overall survival (OS) rate, and the secondary end points were the progression-free survival rate and safety analysis.

Results: Sixty-six patients were randomly assigned to receive wPTX ( $n=33$ ) or wPTX plus VPA ( $\mathrm{n}=33)$. The median OS was 9.8 months in the wPTX group and 8.7 months in the wPTX plus VPA group (hazard ratio $1.19 ; 95 \%$ CI $0.702-2.026 ; P=0.51$ ). The median progression-free survival was 4.5 months in the wPTX group and 3.0 months in the wPTX plus VPA group (hazard ratio $1.29 ; 95 \%$ CI $0.753-2.211 ; P=0.35$ ). Grade $3-4$ adverse events were neutropenia $(3.1 \%)$, pneumonia $(1.6 \%)$, liver injury $(1.6 \%)$, brain infarction $(1.6 \%)$, and rupture of aorta $(1.6 \%)$.

Conclusion: No statistically significant difference was observed between wPTX and wPTX plus VPA for OS.

Keywords: valproic acid, paclitaxel, second- or third-line therapy, advanced gastric cancer

\section{Introduction}

Gastric cancer is a leading cause of cancer death globally. For patients with unresectable advanced or recurrent gastric cancer worldwide, the combination of fluoropyrimidine and platinum is the standard first-line chemotherapy. ${ }^{1}$ Although several randomized studies have verified the survival benefits of second-line chemotherapy compared with best supportive care alone, the median survival was $<6$ months. $^{2-4}$ Therefore, a more active regimen for the second-line treatment is expected.

Although numerous clinical studies have considered the efficacy of molecular targeted agents combined with conventional chemotherapy, their efficacy in gastric cancer was only demonstrated by trastuzumab as a first-line treatment and ramucirumab 
as a second-line treatment. ${ }^{5,6}$ More recently, lapatinib plus paclitaxel (PTX) failed to show efficacy compared to PTX alone as a second-line treatment for the patients with HER2positive gastric cancer. ${ }^{7}$ Other candidates for molecular targeted therapy are needed.

Histone deacetylase (HDAC) inhibitors have antiproliferative effects through cell-cycle arrest, differentiation, and apoptosis in various cancer cell types, including gastric cancer cells. ${ }^{8-10}$ Accordingly, the combination of an HDAC inhibitor with conventional chemotherapy is expected to have a synergistic effect because their mechanism of action varies from those of conventional chemotherapeutic regimens. Valproic acid (VPA), which has long been used clinically to treat epilepsy and bipolar disorder without significant toxic effects, is now also used to prevent migraines. VPA inhibits both class I and II HDACs ${ }^{11}$ and affects tumor growth by inducing p21 WAF1. ${ }^{12,13}$ However, some reports suggest that HDAC inhibitors also enhance the acetylation of nonhistone proteins in relation to apoptosis. ${ }^{14-16}$ Yagi et $\mathrm{al}^{17}$ reported that VPA induced dynamic modulation of histone $\mathrm{H} 3$ and $\alpha$-tubulin acetylation in association with an anticancer effect and the enhancement of PTX in a gastric cancer cell line. Although the efficacy of VPA in human malignancy is unclear, combination therapy with radiation led to improved outcomes in glioblastoma patients. ${ }^{18}$ Therefore, VPA in combination with PTX is a promising therapy for gastric cancer.

Weekly paclitaxel (wPTX) administration of $80 \mathrm{mg} / \mathrm{m}^{2}$ is a second-line treatment option for patients with gastric cancer. ${ }^{19}$ A recent randomized Phase III trial comparing PTX and irinotecan as second-line chemotherapy for gastric cancer found no significant difference in overall survival (OS) between the two groups. Third-line chemotherapy was administered to $89.8 \%$ participants in the PTX group and $72.1 \%$ participants in the irinotecan group. The median OS was 9.5 months for the PTX treatment and 8.4 months for the irinotecan treatment. However, wPTX was associated with a good toxicity profile compared with irinotecan. ${ }^{20}$

We therefore planned a multicenter randomized Phase II study to investigate additional benefits of VPA as a molecular targeting agent with wPTX in second- and third-line chemotherapy. This manuscript is a final report following up to our first paper as the study protocol. ${ }^{21}$

\section{Protocol design \\ Purpose}

This study compared the effects of wPTX alone and wPTX plus VPA in patients with previously treated advanced gastric cancer.

\section{End point}

The primary end point was the OS rate, defined as the time from randomization to death from any cause. The secondary end points were the progression-free survival (PFS) rate, defined as the time from randomization to radiographic progression; response rate; and assessment of peripheral neuropathy in each therapeutic course. The OS rate and PFS rate were estimated according to the Kaplan-Meier method. The response rate was evaluated every two courses during the study and classified based on the Response Evaluation Criteria in Solid Tumors, Version 1.1. Toxicities, including peripheral neuropathy, were graded according to the National Cancer Institute Common Terminology Criteria for Adverse Events, Version 4.0.

\section{Eligibility criteria}

Patients older than 20 years diagnosed with histologically confirmed metastatic or recurrent gastric carcinoma that was unresponsive to first-line or second-line therapy (progressive disease confirmed by imaging studies) were eligible to participate in this study. Other inclusion criteria were the Eastern Cooperative Oncology Group performance status of $0-2$; an interval of at least 4 weeks from the previous treatment; no prior chemotherapy with taxanes; adequate bone marrow, hepatic, and renal functions; and willingness to provide written informed consent.

The exclusion criteria were pregnancy, history of allergy against Cremophor EL, intestinal pneumonia, lung fibrosis, severe chronic obstructive pulmonary disease, coexistence of another malignant neoplasm, psychological disease or brain metastasis, or another severe medical condition.

\section{Treatment methods}

PTX $\left(80 \mathrm{mg} / \mathrm{m}^{2}\right)$ was administered intravenously on days 1,8 , and 15 , every 4 weeks. Thirty minutes before PTX administration, the patients were premedicated with histamine receptor-1 and -2 blockers and dexamethasone for prophylaxis of allergic reactions. VPA was administrated orally everyday at a dose of $7.5 \mathrm{mg} / \mathrm{kg}$ twice daily. In this way, the serum value reached a concentration of $50-100 \mu \mathrm{g} / \mathrm{mL}$, which is near the concentration required for VPA to act as an HDAC inhibitor (0.4-0.7 mM; 66.4-116.2 $\mu \mathrm{g} / \mathrm{mL}){ }^{22}$ The trough values of VPA in plasma were verified after the first cycle.

\section{Study design}

The study was a prospective, multicenter, randomized Phase II clinical trial. As of 2012, the participating institutions included 18 specialized centers. The protocol was approved 
by the independent ethics committee or institutional review board of each participating institution (Kanazawa University Hospital, Kurobe City Hospital, Toyama Rosai Hospital, Yatsuo General Hospital, Toyama Prefectural Central Hospital, Toyama City Hospital, Takaoka City Hospital, Keiju General Hospital, Kanazawa Medical University Hospital, Ishikawa Prefectural Center Hospital, Asanogawa General Hospital, Keiju Kanazawa Hospital, JCHO Kanazawa Hospital, Kanazawa Medical Center Hospital, Kanazawa Red Cross Hospital, Central Hospital of Matto Ishikawa, Houju Memorial Hospital, and Fukui University Hospital). This trial was conducted in accordance with the Declaration of Helsinki. All patients provided written informed consent before entering the study.

After checking eligibility, patients were randomly assigned at a 1:1 ratio to receive VPA or not. Random assignment was carried out at the data center using a minimization method with the following adjustment factors: Eastern Cooperative Oncology Group performance status (0-1 vs 2), prior chemotherapy (first-line vs second-line), and measurable lesions (presence vs absence). Neither investigators nor patients were blinded to the allocated treatment.

\section{Statistical methods}

The reported median OS for patients with advanced gastric cancer treated with wPTX as the second-line chemotherapy in two studies was 5 months and 6.9 months, respectively. ${ }^{19,23}$ For an exploratory study, if the median OS times for wPTX and wPTX plus VPA are reportedly 5.0 months and 8.0 months, respectively, then 31 patients per treatment arm would be required to detect a difference with $80 \%$ power at a $5 \%$ significance level using a one-sided log-rank test of the quality of survival curves. As we assumed a dropout rate of $5 \%$, the number of patients per treatment group was set at 33, with a total sample size of at least 66 patients.

The study protocol was registered in the UMIN Clinical Trial Registry (UMIN000005887) on August 1, 2011.

\section{Results}

Between June 1, 2011, and June 30, 2014, 66 patients were enrolled and randomly assigned to receive wPTX $(n=33)$ or wPTX plus VPA $(n=33)$ in 18 centers of the Digestive Disease Support Organization. One patient in the wPTX group was ineligible due to a bone fracture after assignment, and one patient withdrew consent (Figure 1). The baseline characteristics of patients and tumors were generally well balanced between the groups using a minimization method with the adjustment factors (Table 1). The mean trough value of VPA in plasma was $63.7 \pm 7.2 \mu \mathrm{g} / \mathrm{mL}$, which was the minimum value just before VPA administration.

The median numbers of cycles were 5 (range $0-16$ ) in the wPTX group and 3.5 (range 0-17) in the wPTX plus VPA group. Afterward, third- or fourth-line chemotherapy was administered to five patients $(15.2 \%)$ in the wPTX group and four patients $(12.9 \%)$ in the wPTX plus VPA group.

There were 28 deaths in the wPTX group and 27 deaths in the wPTX plus VPA group. The median OS was 9.8 months in the wPTX group and 8.7 months in the wPTX plus VPA group (hazard ratio $1.19 ; 95 \%$ CI $0.702-2.026 ; P=0.51$ ). The two groups did not significantly differ in OS (Figure 2A). The median PFS was 4.5 months in the wPTX group and 3.0 months in the wPTX plus VPA group (hazard ratio 1.29; 95\% CI 0.753-2.211; $P=0.35)$. The PFS also did not significantly differ between the two groups (Figure $2 \mathrm{~B}$ ). The results

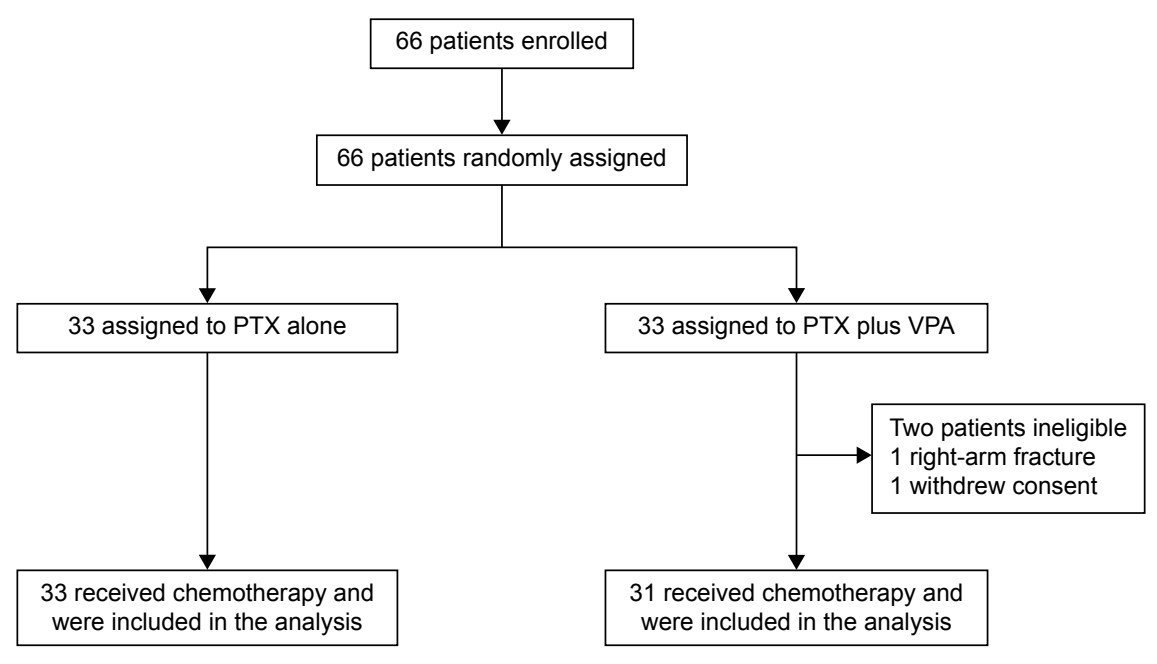

Figure I Trial profile.

Abbreviations: PTX, paclitaxel; VPA, valproic acid. 
Table I Patient characteristics

\begin{tabular}{lll}
\hline Characteristics & $\begin{array}{l}\text { PTX }(\mathbf{n}=\mathbf{3 3}) \\
\text { group }\end{array}$ & $\begin{array}{l}\text { PTX plus VPA (n=33) } \\
\text { group }\end{array}$ \\
\hline $\begin{array}{l}\text { Median age (range), years } \\
\text { ECOG PS, n (\%) }\end{array}$ & $68(49-84)$ & $67(3 \mathrm{I}-83)$ \\
$\quad 0-\mathrm{I}$ & $28(85)$ & $29(88)$ \\
2 & $5(15)$ & $4(12)$ \\
$\begin{array}{l}\text { Prior CTX, n (\%) } \\
\quad \text { First-line }\end{array}$ & $22(67)$ & $20(6 \mathrm{I})$ \\
$\quad$ Second-line & II (33) & $13(39)$ \\
$\begin{array}{l}\text { Target lesion, } n(\%) \\
\quad \text { Yes }\end{array}$ & II (33) & II (33) \\
$\quad$ No & $22(67)$ & $22(67)$ \\
\hline
\end{tabular}

Abbreviations: PTX, paclitaxel; VPA, valproic acid; ECOG PS, Eastern Cooperative Oncology Group performance status; CTX, chemotherapy.

of the subgroup OS analysis are shown in Figure 3. Although treatment with wPTX plus VPA conferred a slight survival advantage in younger $(<65$ years $)$ patients, no significant interactions were observed in any subgroup.

Six patients (9.5\%) suffered adverse events $>$ grade 2 during treatment, including one each with neutropenia, pneumonia, liver injury, brain infarction, and ruptured abdominal aortic aneurysm in the wPTX group and one with pneumonia in the wPTX plus VPA group. There were no special adverse events due to the addition of the VPA.

\section{Discussion}

To our knowledge, this is the first randomized Phase II trial to compare PTX with PTX plus VPA in advanced refractory gastric cancer.

In this study, PTX plus VPA did not provide a survival advantage compared with PTX alone in second- or third-line chemotherapy for advanced gastric cancer.

In previous Phase III studies of WPTX as second-line chemotherapy for gastric cancer, the median OS was
9.5 months and 7.4 months, respectively. ${ }^{6,20}$ In this study, the control wPTX group had a median OS of 9.8 months. Considering that one-third of the control group was treated with third-line chemotherapy, this result might be satisfactory. However, survival of the wPTX plus VPA group was not significantly longer than that of the wPTX-only group. VPA, which is an HDAC inhibitor, induces differentiation and apoptosis of tumor cells. ${ }^{8-10}$ In vitro models that use the OCUM-2MD3 gastric cancer cell line indicated that VPA with PTX decreases growth and promotes apoptosis synergistically. ${ }^{17}$ Considering the amount of VPA administered in this regimen $(15 \mathrm{mg} / \mathrm{kg} / \mathrm{d})$, patients in this study should achieve therapeutic plasma levels between $50 \mu \mathrm{g} / \mathrm{mL}$ and $100 \mu \mathrm{g} / \mathrm{mL} .{ }^{22}$ In this study, the mean trough value of plasma VPA was $63.7 \pm 7.2 \mu \mathrm{g} /$ $\mathrm{mL}$. Because this concentration range was predicted to increase histone acetylation in preclinical models, the idea that this dose of VPA would have no additional effect is not appropriate.

Several single-arm trials have reported potential benefits from adding VPA as combination epigenetic therapy in glioblastoma and myeloid malignancies. ${ }^{18,24-26}$ However, the specific addition of VPA to PTX in this study did not show encouraging results. In a randomized Phase II study of myeloid malignancies to assess the efficacy of VPA in combination with decitabine as a hypomethylating agent, the addition of VPA to decitabine also did not show improved outcome over decitabine alone. ${ }^{27}$ The negative experience may be due to the fact that HDAC inhibitors enhance regulatory T-cell (Treg) function by increasing FOXP3 expression in T cells. ${ }^{28}$ Treg cells play a key role in immune system homeostasis and tolerance to antigens and may be responsible for the lack of an appropriate immune
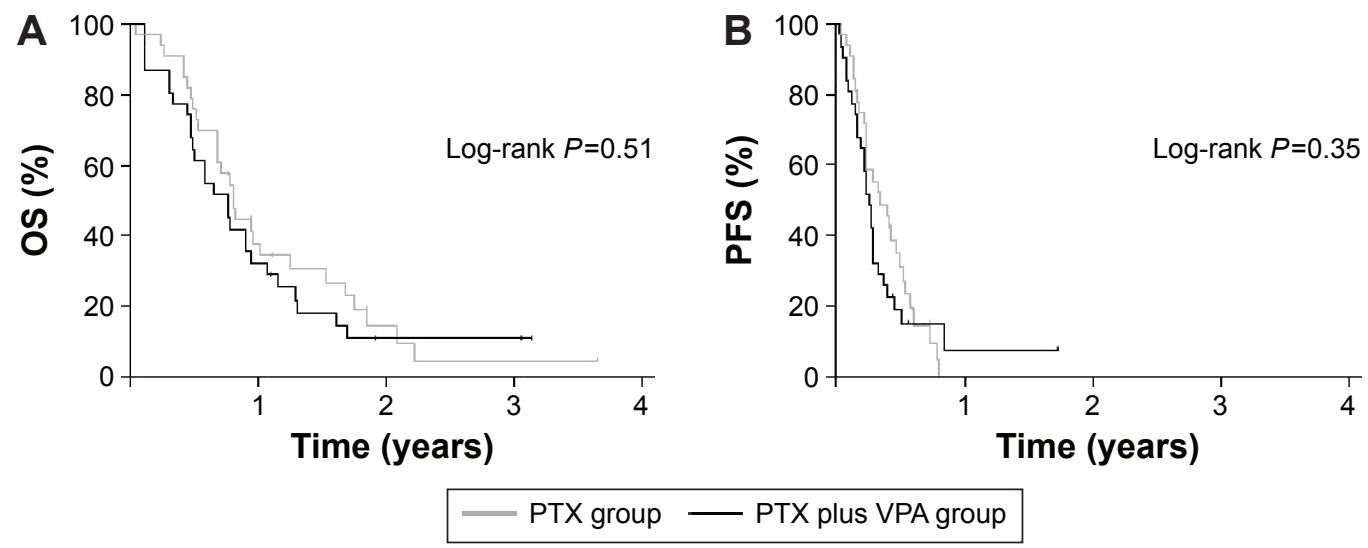

Figure 2 Kaplan-Meier curves of overall survival (A) and progression-free survival (B).

Abbreviations: OS, overall survival; PTX, paclitaxel; VPA, valproic acid; PFS, progression-free survival. 


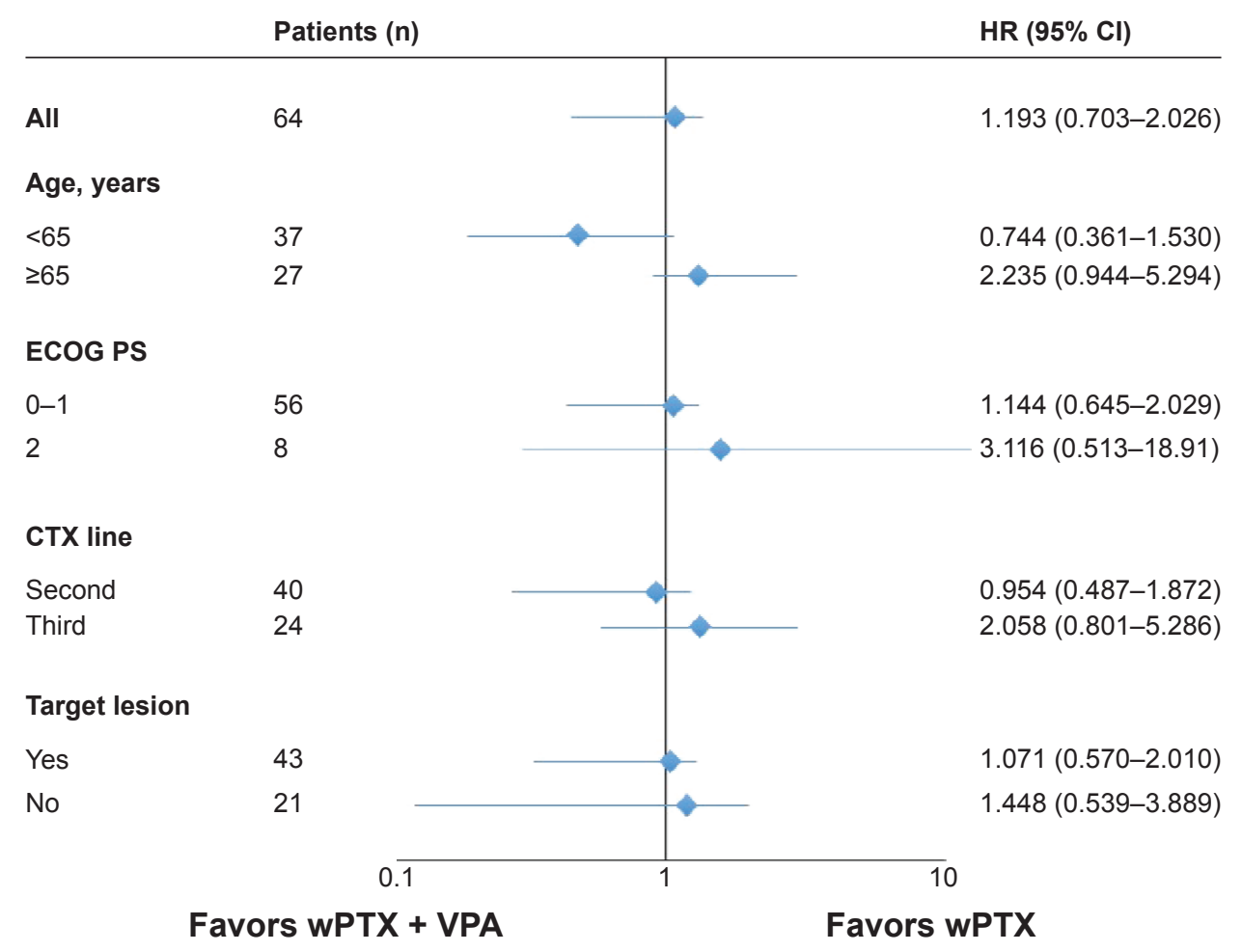

Figure 3 Forest plot of subgroup analyses.

Abbreviations: Cl, confidence interval; HR, hazard ratio; ECOG PS, Eastern Cooperative Oncology Group performance status; CTX, chemotherapy; wPTX, weekly paclitaxel; VPA, valproic acid.

response against tumor cells. In contrast, PTX acts both as an anticancer agent and as an inhibitor of TGF- $\beta$ signaling by blocking of Smad 2/3 phosphorylation. ${ }^{29}$ The cytokine TGF- $\beta$ induces Treg cells from naïve $\mathrm{CD}^{+}, \mathrm{CD} 25^{-} \mathrm{T}$ cells. ${ }^{30,31}$ Treg-cell infiltration into cancer tissue results in immune tolerance and chemoresistance. Therefore, PTX and VPA might actually work against each other. We previously reported that the mean tumor volume of the VPA-treated group was smaller than that of the control group in the xenograft model. However, these models were constructed from nude mice. ${ }^{17}$ Because nude mice lack a thymus, they cannot generate mature lymphocytes, including Treg cells. Thus, VPA treatment might have led to more favorable results than the present study.

\section{Conclusion}

The primary end point of prolonged OS was not met in our study. The clear reason is not known, but subset analysis showed that the wPTX plus VPA regimen may have potential to improve OS in patients younger than 65 years. However, the next randomized Phase III trial comparing wPTX with wPTX plus VPA in patients younger than 65 years may not reach favorable results. Unfortunately, VPA might have limited use in autoimmune disease, allograft, and inflammatory bowel disease, which decrease Treg function.

\section{Acknowledgments}

The authors wish to thank Ms Yukiko Sawamoto for her assistance with data management. They would also like to acknowledge the great contributions of the 18 centers in the Hokuriku region of Japan.

\section{Author contributions}

$\mathrm{SF}, \mathrm{KO}, \mathrm{TF}$, and TO designed the study. SF, JK, MK, YH, and TT gathered the data. SF and JK analyzed and interpreted the data. All authors were involved in the drafting, review, and approval of the manuscript and the decision to submit it for publication.

\section{Disclosure}

The authors report no conflicts of interest in this work.

\section{References}

1. Wagner AD, Grothe W, Haerting J, Kleber G, Grothey A, Fleig WE. Chemotherapy in advanced gastric cancer: a systematic review and meta-analysis based on aggregate data. J Clin Oncol. 2006;24(18):2903-2909.

2. Kang JH, Lee SI, Lim do H, et al. Salvage chemotherapy for pretreated gastric cancer: a randomized phase III trial comparing chemotherapy plus best supportive care with best supportive care alone. J Clin Oncol. 2012;30(13):1513-1518.

3. Ford HE, Marshall A, Bridgewater JA, et al; COUGAR-02 Investigators Docetaxel versus active symptom control for refractory oesophagogastric adenocarcinoma (COUGAR-02): an open-label, phase 3 randomised controlled trial. Lancet Oncol. 2014;15(1):78-86. 
4. Thuss-Patience PC, Kretzshmar A, Bichev D, et al. Survival advantage for irinotecan versus best supportive care as second-line chemotherapy in gastric cancer: a randomised phase III study of the Arbeitsgemeinschaft Internistische Onkologie (AIO). Eur J Cancer. 2011;47(15):2306-2314.

5. Bang YJ, Van Cutsem E, Fetereislova A, et al; ToGA Trial Investigators. Trastuzumab in combination with chemotherapy versus chemotherapy alone for treatment of HER-2 positive advanced gastric or gastrooesophageal junction cancer (ToGA): a phase 3, open-label, randomized controlled trial. Lancet. 2010;376(9742):687-697.

6. Wilke H, Muro K, Cutsem E, et al. Ramucirumab plus paclitaxel versus placebo plus paclitaxel in patients with previously treated advanced gastric or gastro-oesophageal junction adenocarcinoma (RAINBOW): a double-blind, randomized phase 3 trial. Lancet Oncol. 2014;15(11):1224-1235.

7. Satoh T, Xu RH, Chung HC, et al. Lapatinib plus paclitaxel versus paclitaxel alone in the second-line treatment of HER2-amplified advanced gastric cancer in Asian populations: TyTAN - a randomized, phase III study. J Clin Oncol. 2014;32(19):2039-2049.

8. Carey N, La Thangue NB. Histone deacetylase inhibitors: gathering pace. Cur Opin Pharmacol. 2006;6(4):369-375.

9. Suzuki T, Yokozaki H, Kuniyasu H, et al. Effect of trichostatin A on cell growth and expression of cell cycle-and apoptosis-related molecules in human gastric and oral carcinoma cell lines. Int J Cancer. 2000;88(6): 992-997.

10. Zhang X, Yashiro M, Ren J, Hirakawa K. Histone deacetylase inhibitor, trichostatin A, increases the chemosensitivity of anticancer drugs in gastric cancer cell lines. Oncol Rep. 2006;16(3):563-568.

11. Göttlicher M, Minucci S, Zhu P, et al. Valproic acid defines a novel class of HDAC inhibitors inducing differentiation of transformed cells. EMBO J. 2001;20(24):6969-6978.

12. Hrzenjak A, Moinfar F, Kremser ML, et al. Valproate inhibition of histone deacetylase 2 affects differentiation and decreases proliferation of endometrial stromal sarcoma cells. Mol Cancer Ther. 2006;5(9): 2203-2210.

13. Rocchi P, Tonelli R, Camerin C, et al. p21Waf1/Cip1 is a common target induced by short-chain fatty acid HDAC inhibitors (valproic acid, tributyrin and sodium butyrate) in neuroblastoma cells. Oncol Rep. 2005;13(6):1139-1144.

14. Yu X, Guo ZS, Marcu MG, et al. Modulation of p53, ErbB1, ErbB2, and Raf-1 expression in lung cancer cells by depsipeptide FR901228. J Natl Cancer Inst. 2002;94(7):504-513.

15. Blagosklonny MV, Robey R, Sackett DL, et al. Histone deacetylase inhibitors all induce p21 but differentially cause tubulin acetylation, mitotic arrest, and cytotoxicity. Mol Cancer Ther. 2002;1(11): 937-941.

16. Catalano MG, Poli R, Pugliese M, Fortunati N, Boccuzzi G. Valproic acid enhances tubulin acetylation and apoptoic activity of paclitaxel on anaplastic thyroid cancer cell lines. Endocr Relat Cancer. 2007;14(3): 839-845.

17. Yagi Y, Fushida S, Harada S, et al. Effects of valproic acid on the cell cycle and apoptosis through acetylation of histone and tubulin in a scirrhous gastric cancer cell line. J Exp Clin Cancer Res. 2010;29:149.
18. Barker CA, Bishop AJ, Chang M, Beal K, Chan TA. Valproic acid use during radiation therapy for glioblastoma associated with improved survival. Int J Radiat Oncol Biol Phys. 2013;86(3):504-509.

19. Hironaka S, Zenda S, Boku N, Fukutomi A, Yoshino T, Onozawa Y. Weekly paclitaxel as second-line chemotherapy for advanced or recurrent gastric cancer. Gastric Cancer. 2006;9:14-18.

20. Hironaka S, Ueda S, Yasui H, et al. Randomized open-label, phase III study comparing irinotecan with paclitaxel in patients with advanced gastric cancer without severe peritoneal metastasis after failure of prior combination chemotherapy using fluoropyrimidine plus platinum: WJOG 4007 trial. J Clin Oncol. 2013;31(35):4438-4444.

21. Fushida S, Kaji M, Oyama K, et al. Randomized phase II trial of paclitaxel plus valproic acid vs paclitaxel alone as second-line therapy for patients with advanced gastric cancer. Onco Targets Ther. 2015;8: 939-941.

22. Phiel CJ, Zhang F, Huang EY, Guenther MG, Lazar MA, Klein PS. Histone deacetylase is a direct target of valproic acid, a potent anticonvulsant, mood stabilizer, and teratogen. J Biol Chem. 2001;276(39): 36734-36741

23. Arai T, Hamaguchi T, Shirao K. Weekly paclitaxel in patients with heavily treated advanced gastric cancer. Proc Am Soc Clin Oncol. 2003; 22(suppl; abstr 1291):321.

24. Kuendgen A, Strupp C, Aivado M, et al. Treatment of myelodysplastic syndromes with valproic acid alone or in combination with all-trans retinoic acid. Blood. 2004;104:1266-1269.

25. Soriano AO, Yang H, Faderl S, et al. Safety and clinical activity of the combination of 5-azacytidine, valproic acid, and all-trans retinoic acid in acute myeloid leukemia and myelodysplastic syndrome. Blood. 2007;110(7):2302-2308.

26. Gracia-Manero G, Kantarjian HM, Sanchez-Gonzalez B, et al. Phase $1 / 2$ study of the combination of 5-aza-2'-deoxycytidine with valproic acid in patients with leukemia. Blood. 2006;108(10):3271-3279.

27. Issa JP, Gracia-Manero G, Huang X, et al. Results of phase II randomized study of low-dose decitabine with or without valproic acid in patients with myelodysplastic syndrome and acute myelogenous leukemia. Cancer. 2015;121(4):556-561.

28. Fayyad-Kazan H, Rouas R, Merimi M, et al. Valproate treatment of human cord blood CD4-positive effector T cells confers on them the molecular profile (microRNA signature and FOXP3 expression) of natural regulatory $\mathrm{CD} 4$-positive cells through inhibition of histone deacetylase. J Biol Chem. 2010;285(27):20481-20491.

29. Tsukada T, Fushida S, Harada S, et al. Low-dose paclitaxel modulates tumor fibrosis in gastric cancer. Int J Oncol. 2013;42(4):1167-1174.

30. Park HB, Paik DJ, Jang E, Hong S, Youn J. Acquisition of anergic and suppressive activities in transforming growth factor-beta-costimulated CD4+CD25- T cells. Int Immunol. 2004;16(8):1203-1213.

31. Fantini MC, Becker C, Monteleone G, Pallone F, Galle PR, Neurath MF. Cutting edge: TGF-beta induces a regulatory phenotype in CD4+CD25$\mathrm{T}$ cells through Foxp3 induction and down-regulation of Smad7. J Immunol. 2004;172(9):5149-5153.
Drug Design, Development and Therapy

\section{Publish your work in this journal}

Drug Design, Development and Therapy is an international, peerreviewed open-access journal that spans the spectrum of drug design and development through to clinical applications. Clinical outcomes, patient safety, and programs for the development and effective, safe, and sustained use of medicines are a feature of the journal, which
Dovepress

has also been accepted for indexing on PubMed Central. The manuscript management system is completely online and includes a very quick and fair peer-review system, which is all easy to use. Visit http://www.dovepress.com/testimonials.php to read real quotes from published authors. 